\title{
Wavelength Monitoring in DWDM Networks Using Low Cost Semiconductor Laser Diode/Amplifiers
}

\author{
San-Liang Lee, Ching-Tang Pien, and Yu-Yi Hsu \\ Dept. of Electronic Engineering, National Taiwan University of Science and Technology \\ 43, Sec. 4, Keelung Rd., Taipei 106, Taiwan \\ Phone:011-886-2-27376401, Fax:011-886-2-27376424, email:sanlee@et.ntust.edu.tw
}

The rapid increase in the demand of larger channel capacity has driven vast applications of dense wavelength division multiplexing (DWDM) techniques into the broadband information infrastructure. Multiwavelength optical networks with ultrahigh capacity will become feasible only when the wavelengths used in the networks can be properly controlled and monitored. This requires a simple and effective technique to detect or compare wavelengths in the network. Significant efforts on this subject have realized several useful approaches for wavelength stabilization and sensing, see ref. [1-3] and references therein. We proposed a novel technique that uses the detection of the transparent point of a semiconductor optical amplifier (SOA) for wavelength measurement and wavelength tracking [4]. This approach can be monolithically integrated with the semiconductor optoelectronic components used in optical networks, and is thus very attractive for applications in large-scale transmitters and cross-connects. In principle, the transparent condition of a semiconductor gain material is independent of the incident light power and is insensitive to the facet reflection. Thus, a diode laser biased below threshold or a SOA with much relaxed requirements on the antireflection coatings can also be used for this purpose. We report here an empirical investigation on the feasibility of this approach and show that it is viable in photonic networks for wavelength display, wavelength monitoring, and wavelength stabilization.

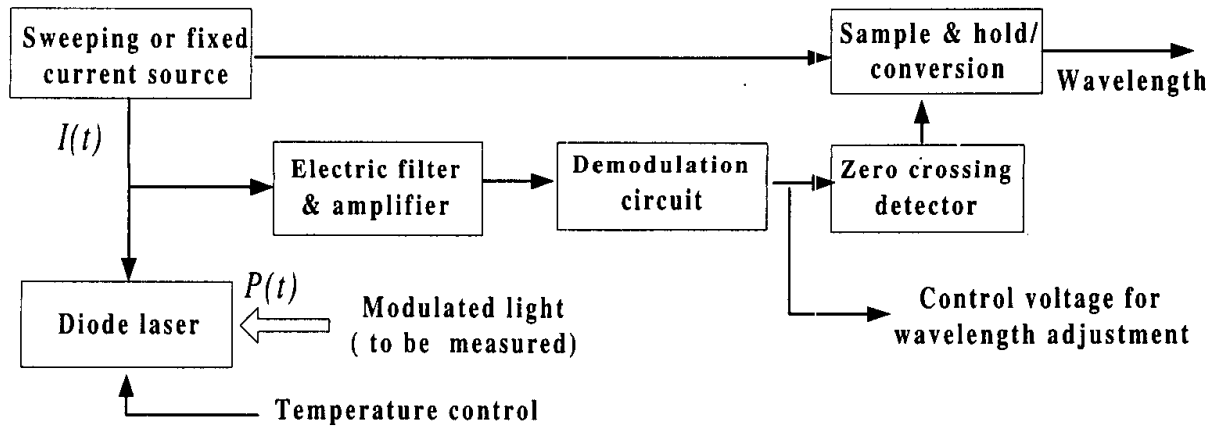

Fig. 1. Schematic of wavelength sensing or stabilization where the laser diode acts as a wavelength discriminator.

Fig. 1 shows the schematic of wavelength sensing or stabilization using a diode laser as a wavelength discriminator. Since a diode laser and a simple detection circuitry are used, they can be integrated into a low-cost and compact module. The transparency corresponds to the condition that the injected carrier density is large enough to make the induced separation between the quasi-Fermi levels of electrons and holes equal the incident photon energy [5], so the transparent current is wavelength dependent. The operation principle of the detection circuit is based on the fact that the induced junction voltage across the diode laser depends on the bias current and the transparent current. During wavelength sensing, the injection current, $I(t)$, to the laser diode is swept over a range that covers the transparent current of the interested wavelengths. The input light is usually modulated with a pilot-tone signal of which the frequency is much larger than the sweeping frequency of the diode current. Since the induced voltage is approximately proportional to $\left(I(t)-I_{t r}\right) P(t)$, where $I_{t r}$ is the transparent current and $P(t)$ is the light power, the transparent current can be detected from zero-crossing point of the demodulated signal of the induced voltage. For wavelength stabilization, the sensor is biased at the transparent current for the incident 
wavelength. When the incident laser wavelength drifts, an induced voltage appears across the electrodes. This voltage is demodulated and fed back to adjust the wavelength of the incident light.

For using a diode laser as a wavelength discriminator, its transparent current was first characterized with a high-precision wavelength meter and a lockin amplifier. The result for a diode laser is shown in Fig. 2 (a). The required injected current to reach transparency is higher for an incident light with a shorter wavelength. The input wavelength must be smaller than the bandgap wavelength and is lower bounded by the condition that its corresponding transparent current must be below the threshold condition. For measuring the data in Fig.1, the light from a tunable laser was modulated and coupled into the diode laser. Since the transparent condition is sensitive to temperature fluctuation, the laser was attached to a copper plate for temperature control during the measurement. The light with an unknown wavelength can then be determined from comparing the measured transparent current to the above data.

(a)

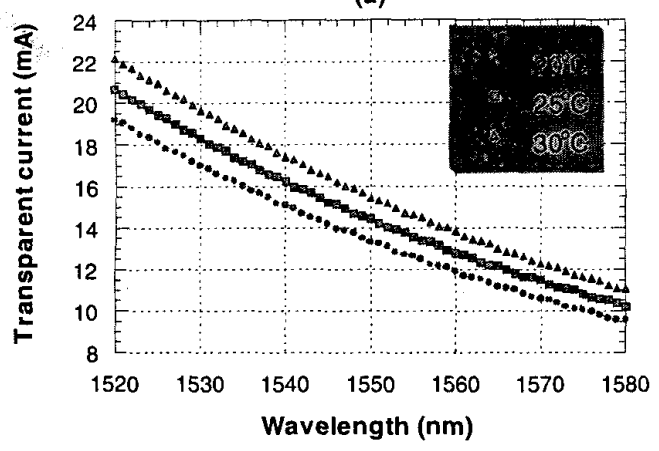

(b)

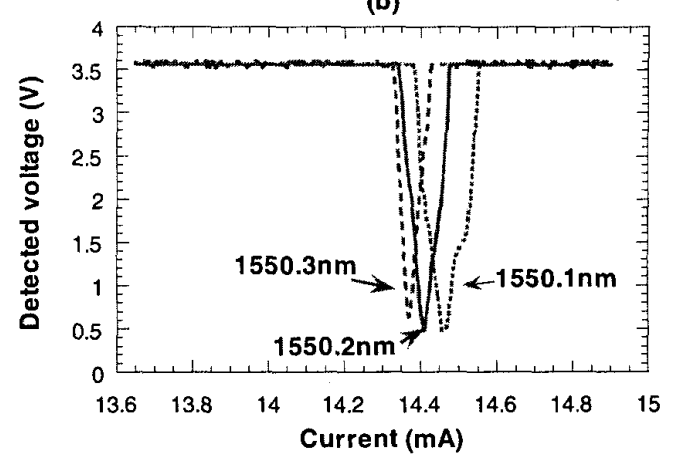

Fig. 2. (a) variation of transparent current with the wavelength and (b) detected voltage (after the demodulated circuit) versus the swept current for three wavelengths spaced by $0.1 \mathrm{~nm}$

The transparent points for three wavelengths that differ in $0.1 \mathrm{~nm}$ can be clearly observed in Fig. 2(b), so a sub-angstrom wavelength resolution that meets the requirement of DWDM systems can be achieved with this approach. In the figure, the detected voltage was obtained after the demodulation circuit and passing through a voltage limiter to manifest the transparent point, which corresponds to the current value at minimal voltage. The error in wavelength sensing is proportional to the error in measuring the transparent current, which results from the fluctuations associated with the driving current, detected voltage, and temperature, respectively. A larger slope for the curves in Fig. 2(a) will give rise to a higher resolution in wavelength sensing; and a small temperature variation rate for transparent current will relax the requirement on temperature control. For example, the slope at $25^{\circ} \mathrm{C}$ is about $-0.2 \mathrm{~mA} / \mathrm{nm}$ at 1550 $\mathrm{nm}$ wavelength. The slope is higher when the temperature increases. A larger transparent current results in a larger slope, and thus a higher wavelength resolution. The error in measuring the transparent current must be kept within $2 \mu \mathrm{A}$ to achieve $0.01 \mathrm{~nm}$ wavelength accuracy. The resolution of wavelength measurement is also limited by the noise associated with the transparency detection. The fluctuation in the detected voltage comes from the shot noise in detected process and the thermal noise from the series and load resistance. To minimize the noise, a single-frequency pilot tone was applied to modulate the incident light, and a sufficiently narrow bandpass filter was used to reject most of the noise.

For wavelength stabilization, one would concern how sensitive the induced voltage is with respect to a wavelength drift. Fig. 3 shows the induced voltage (before amplification) for three different bias currents when the wavelength varies. The slope is around $50 \mu \mathrm{V} / \mathrm{nm}$ for all the curves. In this measurement the light power coupled into the laser diode was estimated to be $0.5 \mathrm{~mW}$. Higher input powers will result in larger slope and higher sensitivity to a given wavelength drift. The induced voltage will go up by $0.5 \mu \mathrm{V}$ for $0.01 \mathrm{~nm}$ of wavelength change. This voltage change can be easily detected with the amplifying and filtering circuits shown in Fig. 1. 


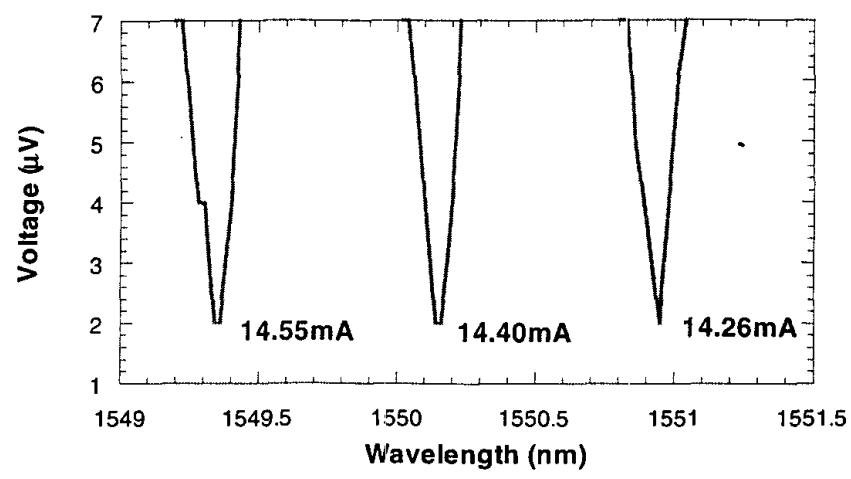

Fig. 3. Variation of the induced voltage across the laser electrodes with wavelength change for three different bias currents.

Another critical issue for wavelength stabilization is its stability. Since the transparency is a unique property for direct-bandgap optoelectronic devices, it should not change with time if the device temperature can be fixed. Furthermore, because of its low biasing current, comparing to a diode laser operated above threshold, the degradation problem should be of less concern. Thus wavelength monitoring using transparency detection should be very stable and reliable, for both discrete and integrated devices. Fig. 4 shows that the induced voltage varies less than $0.25 \mu \mathrm{V}$ over 33 hours of continuous measurement when the input wavelength is fixed. Refer to Fig. 2 and 3, this variation indicates about $0.005-\mathrm{nm}$ change in the input wavelength or $1.0-\mu \mathrm{A}$ shift in the transparent current. This variation is negligible for most DWDM applications. Also shown in the figure is the input wavelength fluctuation measured with a commercial wavelength meter. Noting that the wavelength varies at most $0.0035 \mathrm{~nm}$ during the measurement that is close to the value implied by the voltage variation.

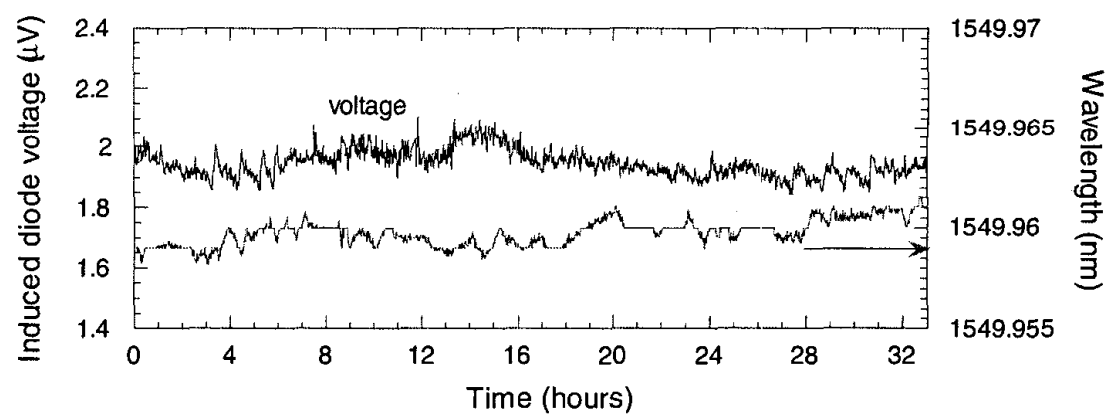

Fig. 4. Induced diode voltage for 24 hours of continuous running when the bias was fixed at the transparency

In summary, we addressed some critical issues of using a low-cost laser diode as a wavelength discriminator for applications in DWDM systems, and showed that sub-angstrom wavelength resolution can be achieved. For wavelength stabilization, this approach has very good sensitivity to the wavelength drift. We also demonstrated that the transparent property of a diode laser is stable and reliable.

\section{REFERENCES}

1. T. Ikegami, S. Sudo, and Y. Sakai, Frequency Stabilization of Semiconductor Laser Diodes, (Boston, MA: Artech House, 1995) chap. 5

2. L.E. Nelson, S.T. Cundiff, and C.R. Giles,"Optical monitoring using data correlation for WDM systems," IEEE Photon. Technol. Lett., 10, 1030-1032, 1998.

3. T. Coroy, R.M. Measures, T.H. Wood, and C.A. Burrus, "Active wavelength measurement system using an InGaAsP-InP quantum well electroabsorption filtering detector," IEEE Photon. Technol. Lett., 8, 1686-1688, 1996

4. S.-L. Lee, "Wavelength measurement and Tracking Using Semiconductor Laser Amplifiers for Applications in Photonic Networks," IEEE Photon. Technol. Lett., 10, 439-441, 1998.

5. L.A. Coldren and S.W. Corzine, Diode Lasers and Photonic Integrated Circuits, (John Wiley \& Sons, 1995) Chap. 5. 